Enferm Bras 2019;18(5):710-20

https://doi.org/10.33233/eb.v18i5.2844

\title{
REVISÃo \\ Contributos da música na reabilitação da pessoa após acidente vascular cerebral
}

Raquel Alexandra Teixeira da Silva*, Olga Maria Pimenta Lopes Ribeiro**, Hugo Filipe dos Santos Neves ${ }^{* *}$

*Enfermeira no Centro Hospitalar São João, Mestranda em Enfermagem de Reabilitação na Escola Superior de Saúde de Santa Maria (ESSSM), Portugal, **Professora adjunta na Escola Superior de Enfermagem do Porto, Investigadora integrada no Centro de Investigação em Tecnologias e Serviços de Saúde (CINTESIS), ${ }^{* * * E n f e r m e i r o ~ n o ~ C e n t r o ~ H o s p i t a l a r ~ S a ̃ o ~ J o a ̃ o, ~}$ Especialista em Enfermagem de Reabilitação

Recebido 29 de abril de 2019; aceito 15 de setembro de 2019.

Correspondência: Raquel Alexandra Teixeira da Silva, Escola Superior de Saúde de Santa MariaTv. de Antero de Quental 173 175, 4049-024 Porto, Portugal

Raquel Alexandra Teixeira da Silva: raquelalexsilva.silva@gmail.com

Olga Maria Pimenta Lopes Ribeiro: olgaribeiro25@hotmail.com

Hugo Filipe dos Santos Neves: hugoneves1988@gmail.com

\section{Resumo}

Objetivo: Determinar os contributos da música na reabilitação da pessoa após acidente vascular cerebral. Métodos: Trata-se de uma revisão integrativa, cuja colheita de dados foi realizada em abril de 2019 nas bases de dados Medline, Cinahl e Pubmed. Resultados: A análise dos 12 artigos incluídos no estudo permitiu identificar várias intervenções com recurso à música que obtiveram como resultado melhorias a diversos níveis entre eles, nomeadamente na marcha, força, equilíbrio, habilidades motoras, humor e comunicação. Conclusão: As evidências demonstram que o desenvolvimento e a implementação de programas de reabilitação com recurso à música contribuem para a recuperação da pessoa após AVC.

Palavras-chave: musicoterapia, música, acidente vascular cerebral, reabilitação.

\section{Abstract}

\section{Contributions of music in the rehabilitation of people after stroke}

Objective: To determine the contributions of music in the rehabilitation of people after stroke. Methods: This is an integrative review collected in April 2019 from the Medline, Cinahl and Pubmed databases. Results: The analysis of the 12 articles included in the study allowed us to identify various interventions using music that resulted in improvements at various levels, including gait, strength, balance, motor skills, humor and communication. Conclusion: Evidence shows that the development and implementation of rehabilitation programs using music contribute to the recovery of the person after stroke.

Key-words: music therapy, music, stroke, rehabilitation.

\footnotetext{
Resumen

Contribuciones de la música en la rehabilitación de personas después de un accidente cerebrovascular

Objetivo: Determinar las contribuciones de la música en la rehabilitación de personas después de un accidente cerebrovascular. Métodos: Esta es una revisión integradora, que se recopiló en abril de 2019 de las bases de datos Medline, Cinahl y Pubmed. Resultados: El análisis de los 12 artículos incluidos en el estudio identificó varias intervenciones usando música que dieron como resultado mejoras en varios niveles, incluyendo marcha, fuerza, equilibrio, habilidades motoras, humor y comunicación. Conclusión: La evidencia muestra que el desarrollo y la implementación de programas de rehabilitación que usan música contribuyen a la recuperación de la persona después del accidente cerebrovascular.
}

Palabras-clave: musicoterapia, música, accidente cerebrovascular, rehabilitación. 
O Acidente Vascular Cerebral (AVC) é caracterizado por uma interrupção sanguínea a nível cerebral, podendo esta ser causada pela existência de coágulos (AVC isquémico) ou até mesmo rotura do vaso (AVC hemorrágico). Determinado pelo défice de nutrientes e oxigénio, na região afetada, o AVC pode provocar várias manifestações influenciadas pelo tipo, local e extensão da lesão [1].

De facto, apesar de a sintomatologia ser variável, podemos afirmar que o AVC, de modo generalizado, poderá provocar défices a vários níveis: consciência, visão, fala, linguagem, força, sensibilidade, equilíbrio, marcha, incontinência de esfíncteres, etc. [2]. A pessoa, após AVC, vivência diversos graus de incapacidade e morbilidade, com consequente alteração do quotidiano da pessoa e família, com repercussões a nível financeiro e sobrecarga familiar [3].

O trauma psicológico é também descrito como uma consequência do AVC, em que a pessoa experimenta sentimentos negativos, entre eles a ansiedade, alterações do humor, medo, frustração e tristeza [4].

O AVC, pelas suas manifestações e consequências, é considerado a principal causa de incapacidade a longo prazo a nível mundial [5]. Estima-se que cerca de $80 \%$ da carga econômica de doenças cerebrais é devido a custos de tratamento e respetivos cuidados [6]. Razões estas que reforçam a necessidade de avanços científicos na recuperação da pessoa após AVC.

O início de um programa de reabilitação o mais precoce possível é crucial na recuperação após AVC, devendo envolver a família e uma equipe multidisciplinar, facilitando assim a transição para a nova condição de vida [7]. Alguns autores defendem que na fase aguda do AVC os ganhos funcionais advêm de um processo espontâneo de recuperação, associado à redução do edema cerebral, absorção no tecido lesado e aumento do fluxo sanguíneo [8]. Porém, vários investigadores comprovam, através de estudos experimentais, que a reabilitação na fase crônica do AVC é um desafio que permite alcançar ganhos em saúde, nomeadamente a nível motor e cognitivo $[9,10]$. Um estudo de grande escala, realizado nos Estados Unidos da América, revela que poderão existir melhorias funcionais até um ano após AVC [7].

A música é, historicamente, vista como uma forma de expressão do ser humano e desde o século $\mathrm{V}$ a.C. que existe conhecimento de que a música afeta a saúde e o bem-estar [11]. Todavia, apenas na II Guerra Mundial, nos Estados Unidos da América, é que a música foi utilizada em contexto hospitalar com o objetivo de relaxar e proporcionar efeitos sedativos aos feridos, surgindo assim a necessidade de investigar sobre os benefícios da música [12].

A utilização da música por profissionais de saúde com fim terapêutico tem permitido identificar diversos benefícios, porém denota-se uma grande heterogeneidade no método utilizado e na intervenção escolhida [12]. Considerando que até o nível da nomeação da intervenção com recurso à música existem diferenças, para garantir a uniformização emergiu a necessidade de identificar os tipos de intervenções existentes, bem como a sua definição.

Tabela I - Intervenções terapêuticas com recurso à música.

Musicoterapia Intervenção baseada em música em ambiente clínico, por um musicoterapeuta, com recurso a vários

elementos musicais (ex. ouvir música, cantar...)

Intervenção baseada na música $\quad$ Refere-se a todos os protocolos experimentais que utilizam a música para estudar os seus efeitos terapêuticos

\begin{tabular}{ll}
\hline Medicina musical & $\begin{array}{l}\text { Quando um profissional de saúde utiliza intervenções } \\
\text { baseadas na música para a promoção de saúde }\end{array}$ \\
\hline Estimulação auditiva e rítmica & $\begin{array}{l}\text { São apresentados estímulos auditivos a um ritmo fixo e os } \\
\text { movimentos corporais são sincronizados, tendo por objetivo } \\
\text { reabilitar movimentos que são naturalmente rítmicos (ex. } \\
\text { marcha) }\end{array}$ \\
\hline Terapia com suporte musical & $\begin{array}{l}\text { Quando são utilizados instrumentos musicais na reabilitação } \\
\text { motora, treinando motricidade fina e grossa }\end{array}$ \\
\hline Terapia de entonação melódica & $\begin{array}{l}\text { Intervenção baseada no canto desenvolvida para reabilitar } \\
\text { perturbações da fala. }\end{array}$ \\
\hline
\end{tabular}

Fonte: Sihvonen et al. [6].

Partindo do princípio da neuroplasticidade foram efetuados diversos estudos experimentais que comprovam benefícios da música na reabilitação neurológica, nomeadamente: melhorias cognitivas e na comunicação; ganhos na força, equilíbrio e marcha; 
diminuição do grau de dependência; melhoria na qualidade de vida e melhoria do estado emocional [8].

Neste sentido e atendendo ao potencial de recuperação é espectável que o Enfermeiro Especialista em Enfermagem de Reabilitação (EEER) foque a sua ação na manutenção e promoção do bem-estar e qualidade de vida, na melhoria da funcionalidade, maximização das capacidades e prevenção de complicações [13], permitindo que o processo de transição ocorra de forma saudável e consciente [14].

Dada a relevância dos fatos acima descritos e de modo a justificar a pertinência da música nos programas de reabilitação, considerou-se imperativo reunir a evidência existente sobre os contributos da música na reabilitação da pessoa após AVC.

\section{Material e métodos}

Com vista a sintetizar o conhecimento existente sobre o contributo da música na reabilitação da pessoa após AVC considerou-se relevante efetuar uma revisão integrativa sobre o tema.

Assim, seguindo a metodologia PICO para a realização desta revisão integrativa foi efetuada uma pesquisa nas bases de dados: Cinahl, Medline e Pubmed. Para tal, foi tido como foco as pessoas após AVC (participantes), as intervenções com musicoterapia (tipo de intervenção(s)/ fenômenos de interesse) e os resultados relativos à eficácia da implementação das intervenções com musicoterapia, num programa de reabilitação (outcomes).

Os descritores utilizados para a pesquisa foram: Stroke, Music Therapy, Rehabilitation, Music e Stroke Rehabilitation. Relativamente às frases a boleanas, na base de dados Cinahl® foi: ((MH "Stroke+") AND (MM "Music Therapy") AND (MH "Rehabilitation+")); na Medline®: ((MH "Stroke+") AND ((MM "Music Therapy") OR (MM "Music")) AND ((MM "Stroke Rehabilitation") OR $(\mathrm{MH}$ "Rehabilitation+"))) ; na Pubmed®: ((music therapy) AND stroke) AND rehabilitation.

A pesquisa e identificação dos estudos a incluir na revisão integrativa da literatura foi realizada por dois revisores, de forma independente, a 4 de abril de 2019. A triagem foi concretizada por meio da validação dos critérios inseridos na questão de investigação: participantes, intervenção e resultados.

Para a seleção dos estudos e tendo como objetivo identificar a evidência atual, foi considerado o período de publicação compreendido entre 1 de janeiro de 2017 e 4 de abril de 2019. Foram incluídos artigos em inglês, português e espanhol, com referências disponíveis em texto integral.

Os dados foram extraídos por dois revisores com consenso de um outro, de forma independente, após construção de um instrumento de extração de dados [15] que incluiu informações sobre: autores, ano, país onde foi implementado o estudo, características dos participantes, tipo de estudo, intervenções e resultados obtidos.

\section{Resultados e discussão}

Tal como explanado na figura 1, da pesquisa suprarreferida resultaram 68 artigos: $20 \mathrm{da}$ base de dados Cinahl, 13 da base de dados Medline e 35 da base de dados Pubmed. Com apoio do Endnote, e após compilação dos artigos mencionados anteriormente, verificaram-se 12 artigos duplicados. Após leitura de título e abstract foram excluídos 32 artigos, visto não contribuírem para a resposta à pergunta de investigação definida. Porém, dos 24 artigos, foram utilizados para a revisão 13 estudos por estarem disponíveis em completo integral. $\mathrm{Na}$ consequente leitura dos artigos foi excluído um, por informar que o seu conteúdo não poderia ser apresentado ou relatado por outros investigadores que não os próprios autores do estudo.

Os 12 estudos selecionados foram avaliados metodologicamente, após aplicação da Checklist for Cohort Studies, Checklist for Quasi-Experimental Studies e Checklist Randomized Controlled Trial of Joanna Briggs Institute, tendo integrado a revisão integrativa por cumprirem os critérios de avaliação metodológica previamente definidos [15].

Os estudos incluídos nesta revisão são oriundos de diversos países, focando duas publicações na Suécia, Estados Unidos da América e França e uma publicação em Itália, Grécia, Coreia do Sul, Alemanha, Inglaterra e Bélgica. Importa referir que em todos os estudos foram respeitados os critérios éticos referentes à investigação realizada. 


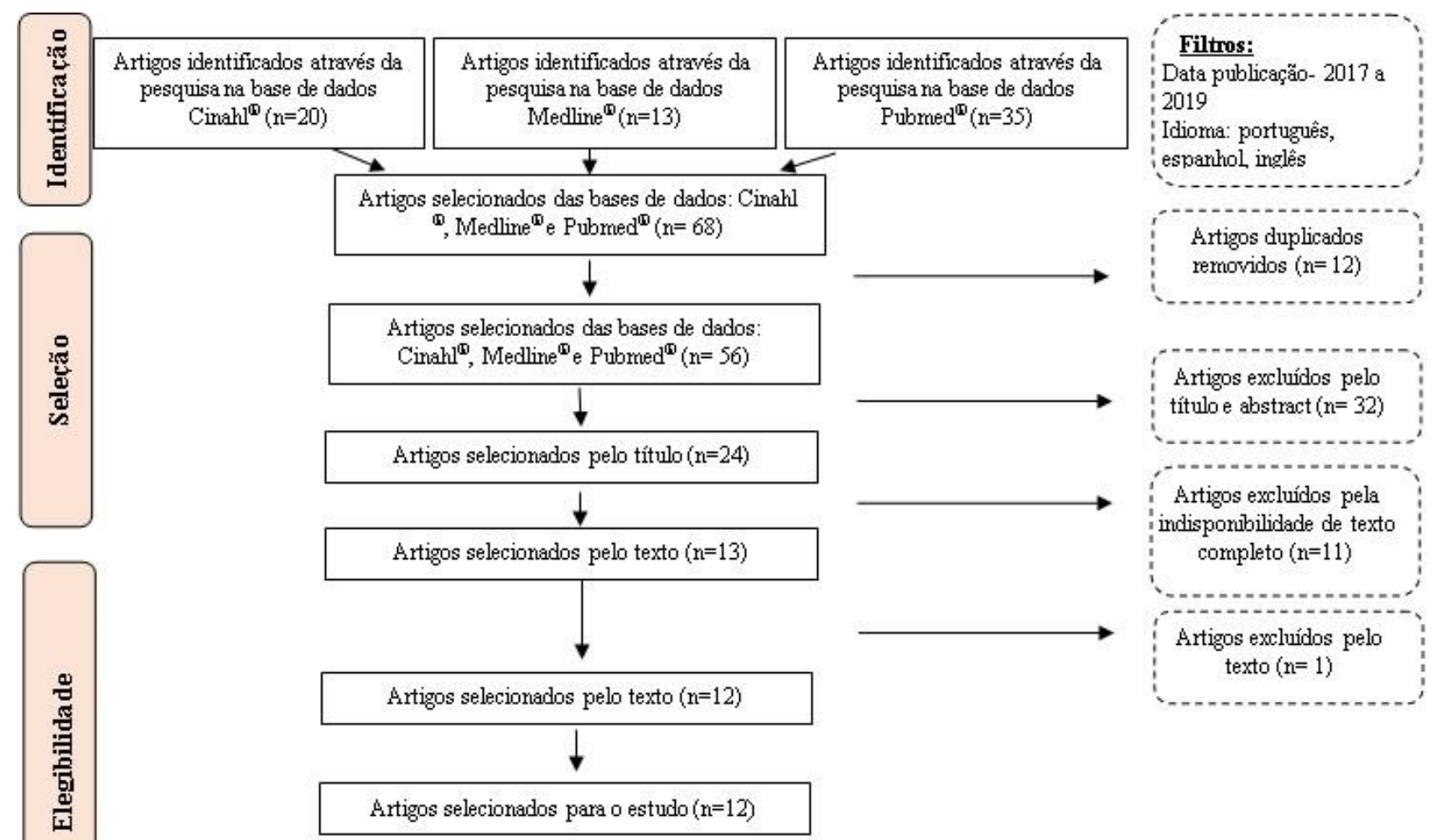

Fonte: os autores

Figura 1 - Diagrama do processo de seleção.

$\mathrm{Na}$ tabela II são apresentados, de forma sucinta e esquematizada, os estudos que compõe esta Revisão, e nela constam: os autores dos estudos, o ano de publicação, o país, as características dos participantes, as intervenções e os resultados dos estudos incluídos.

Tabela II - Sumarização dos estudos incluídos na revisão. (ver PDF em anexo)

No decurso da leitura, análise e avaliação dos 12 artigos que constituem esta revisão verificou-se uma grande heterogeneidade dos mesmos, no que concerne ao tipo de estudo, às características da população, bem como às intervenções implementadas e ao período de implementação. No entanto, verifica-se que os dois estudos realizados na Suécia, em 2017 e 2018, o desenho de estudo é o mesmo.

Embora a diversidade de estudos seja uma característica evidente, existem fatores comuns nos 12 artigos selecionados, o que permite dar resposta à pergunta de investigação proposta para esta revisão. Todos os artigos abordam os benefícios e/ ou contributos da música num programa de reabilitação na pessoa após AVC.

Dando enfoque às intervenções que constituem os programas de reabilitação estudados nos artigos primários supracitados, verifica-se que a estimulação auditiva e rítmica foi a mais utilizada $[17,18,21,23,26]$, seguindo-se da terapia com suporte musical $[16,18,19,27]$, a terapia de entonação melódica [21] e a musicoterapia [25]. Nas duas revisões foram incluídos estudos primários com intervenção da musicoterapia, terapia de entonação melódica, terapia com suporte musical e estimulação auditiva rítmica.

Nos estudos primários que experimentaram a estimulação auditiva e rítmica variou 0 método bem como o tempo de intervenção. Nos cinco artigos constam os seguintes métodos: os participantes ouviam música enquanto executavam um ritmo coordenado, movimentos corporais (metodologia mais utilizada); num outro estudo as pessoas ouviam música enquanto caminhavam em cima de uma esteira. O tempo de implementação dos programas de reabilitação variaram entre 4 semanas e 6 meses, havendo estudos que abordavam o grupo de exercício duas vezes por semana e outros entre 4 e 5 . Todas as sessões eram realizadas em grupo, com 
durabilidade variando entre 15 e 90 minutos. Apesar de alguma diversidade entre os estudos, conclui-se, de um modo genérico, que houve melhorias em nível motor e cognitivo, e a melhoria da marcha foi descrita em 4 estudos, havendo ainda registos de melhoria do equilíbrio e da força $[17,18,21,23,26]$.

Nos estudos experimentais em que a intervenção foi a terapia com suporte musical utilizou-se maioritariamente o piano, seguindo-se da bateria, instrumentos de percussão e iPads $\AA$. O objetivo da utilização dos instrumentos não era apenas reproduzir músicas, era essencialmente seguir uma sequência/ esquema criado pelo investigador. $O$ intervalo de tempo de implementação dos programas variou entre 8 e 18 semanas, com sessões entre 2 e 3 vezes por semana, em que cada uma poderia durar entre 20 minutos e 1 hora. Os resultados obtidos não são todos consensuais, no entanto são descritos ganhos nas habilidades motoras, cognitivas, melhorias na comunicação e no desempenho das Atividades de Vida Diárias (AVDs) $[16,19,20,27]$.

No que diz respeito à terapia de entonação melódica, foi inserida num programa de reabilitação em simultâneo com a estimulação auditiva e rítmica. Os participantes eram incentivados a cantar sentados e enquanto andavam. Não sendo possível saber os resultados obtidos através da terapia de entonação melódica, isoladamente, podemos afirmar que através do programa instituído foi possível melhorar a marcha [21].

A musicoterapia num dos artigos selecionados para a revisão, foi aplicada concomitantemente com um programa de reabilitação convencional, num período de 2 meses, duas vezes por semana com uma duração de 40 minutos por sessão. Os participantes do grupo afirmaram que a musicoterapia influencia positivamente os seus sentimentos, descrevendo após a sessão como "relaxado", "leve" e "descansado". Quando questionados os cuidadores, para além de unanimemente acreditarem na viabilidade da musicoterapia e nos seus benefícios para os participantes, $53 \%$ visualizaram melhorias no humor, na motivação, na autoestima, na expressão oral e no comportamento [25].

Os resultados obtidos nos estudos primários, publicados de 2017 a 2019, selecionados para esta revisão, corroboram os mencionados nas duas revisões incluídas no estudo, pelo que é possível concluir que a música quando inserida num programa de reabilitação influencia positivamente o participante, obtendo-se ganhos em saúde.

Refletindo no impacto que o AVC tem a nível pessoal, econômico e social é notória a necessidade iminente de prosseguir na procura de novos métodos de reabilitação eficazes.

Com esta revisão foi possível compilar o conhecimento existente sobre os contributos da música na reabilitação da pessoa após AVC, identificando-se benefícios a diversos níveis, nomeadamente: na marcha, na força muscular, no equilíbrio corporal, nas habilidades motoras e cognitivas, no humor e comunicação; com consequentes repercussões na qualidade de vida e independência da pessoa.

Assim sendo, as evidências demonstram que o desenvolvimento e a implementação de programas de reabilitação com recurso à música contribuem para a recuperação da pessoa após AVC. Considera-se, então, imprescindível investir neste método para obter ganhos na reabilitação da pessoa após AVC.

Apesar dos investigadores dos estudos selecionados não serem Enfermeiros Especialistas de Reabilitação, no contexto português, esta revisão integrativa traz como principal contribuição à prática dos enfermeiros especialistas em enfermagem de reabilitação o conhecimento recente dos contributos da música na reabilitação do doente após AVC, justificando a sua integração nos programas de reabilitação concebidos e implementados por estes profissionais.

\section{Referências}

1. OMS. Stroke, Cerebrovascular accident. [citado 2019 Abr 10]. Disponível em: https://www.who.int/topics/cerebrovascular accident/en/

2. Jauch EC, Kasab SA, Stettler B [homepage na internet]. Ischemic Stroke Clinical Presentation [citado 2019 Abr 12]. Disponível em: https://emedicine.medscape.com/article/1916852-clinical\#b3 
3. Mendes K, Silveira R, Galvão C. Revisão Integrativa: método de pesquisa para a incorporação de evidências na saúde e na enfermagem. Texto Contexto Enferm 2008;17:758-64. https://doi.org/10.1590/S0104-07072008000400018

4. Silva E. Reabilitação após o AVC. Faculdade de Medicina da Universidade do Porto 2010. [citado 2019 Abr 11] Disponível em: https://repositorioaberto.up.pt/bitstream/10216/52151/2/Reabilitao\%20aps\%200\%20AVC.pdf

5. Benjamin E, Virani S, Callaway C, Chamberlain A, Chang A. Heart disease and stroke statistics - 2018 Update: a report from the American Heart Association. Circulation 2018;137(12):e67-e492. https://doi.org/10.1161/CIR.0000000000000558

6. Sihvonen A, Särkämö T, Leo V, Tervaniemi M, Altenmüller E, Soinila S. Music-based interventions in neurological rehabilitation. Lancet Neurol 2017;16:648-60. https://doi.org/10.1016/S1474-4422(17)30168-0

7. NINDS. Post-stroke rehabilitation fact sheet. [citado 2019 Abr 11]. https://www.ninds.nih.gov/Disorders/Patient-Caregiver-Education/Fact-Sheets/PostStroke-Rehabilitation-Fact-Sheet\#when

8. Barbosa M. Custos e efetividade da reabilitação após acidente vascular cerebral - uma revisão sistemática. [Dissertação]. Coimbra: Faculdade de Economia da Universidade de Coimbra; 2012. Disponível em:

https://estudogeral.sib.uc.pt/bitstream/10316/21495/1/Disserta\%C3\%A7\%C3\%A3o MG ES Teresa\%20Juli\%C3\%A30 2012.pdf

9. Ripollés P, Rojo N, Grau-Sánchez J , Amengual JL, Càmara E, Marco-Pallarés J. Music supported therapy promotes motor plasticity in individuals. Brain Imaging and Behavior 2015;10:1289-1307. https://doi.org/10.1007/s11682-015-9498-x

10. Cha $Y$, Kim $Y$, Hwang S, Chung $Y$. Intensive gait training with rhythmic auditory stimulation in individuals with chronic hemiparetic stroke: a pilot randomized controlled study. Neuro Rehabilitation 2014;35(4):681-88. https://doi.org/10.3233 / NRE-141182

11. Areias J. A música, a saúde e o bem-estar. Nascer e Crescer 2016;25(1). Disponível em: http://www.scielo.mec.pt/scielo.php?script=sci arttext\&pid=S087207542016000100001

12. Baranow A. Musicoterapia: uma visão geral. São Paulo: Enelivros; 1999.

13. Ordem dos Enfermeiros. Regulamento das Competências Específicas do Enfermeiro. 2010. Disponível em: https://www.ordemenfermeiros.pt/arquivo/legislacao/Documents/LegislacaoOE/Regula mentoCompetenciasReabilitacao aprovadoAG20Nov2010.pdf

14. Meleis A. Transitions theory: Middle-Range and situation specific theories in nursing and practice. New York: Springer Publishing Company; 2010.

15. JBI. Joanna Briggs Institute reviewers' manual: 2014 edition. The Joanna Briggs Institute. 2014. Disponível em: https://nursing.Isuhsc.edu/JBI/docs/ReviewersManuals/Economic.pdf

16. Bernardi NF, Cioffi MC, Ronchi R, Maravita A, Bricolo E, Zigiotto L et al. Improving left spatial neglect through music scale playing. J Neuropsychol 2017;11(1):135-58. https://doi.org/10.1111/inp.12078

17. Bunketorp-Käll L, Lundgren-Nilsson Å, Samuelsson H, Pekny T, Blomvé K, Pekna M et al. Long-term improvements after multimodal rehabilitation in late phase after stroke: a randomized controlled trial. Stroke 2017;48(7): 1916-24. https://doi.org/10.1161/STROKEAHA.116.016433

18. Fotakopoulos $\mathrm{G}$, Kotlia $\mathrm{P}$. The value of exercise rehabilitation program accompanied by experiential music for recovery of cognitive and motor skills in stroke patients. J Stroke Cerebrovasc Dis 2018;27(11):2932-39. https://doi.org/ 10.1016/ j.jstrokecerebrovasdis.2018.06.025

19. Fujioka T, Dawson DR, Wright R, Honjo K, Chen JL, Chen JJ et al. The effects of music-supported therapy on motor, cognitive, and psychosocial functions in chronic stroke. Ann NY Acad Sci 2018;1423(1):264-74. https://doi.org/10.1111/nyas.13706

20. Guilbert A, Clément S, Moroni C. A rehabilitation program based on music practice for patients with unilateral spatial neglect: a single-case study. Neurocase 2017;23(1):1221. https://doi.org/10.1080/13554794.2016.1265652 
21. Lee SY, Seok H, Kim SH, Parque M, Kim J. Immediate effects of mental singing while walking on gait disturbance in hemiplegic stroke patients: a feasibility study. Ann Rehabil Med 2017;42(1):1-7. https://doi.org/10.5535/arm.2018.42.1.1

22. Magee WL, Clark I, Tamplin J, Bradt J. Music interventions for acquired brain injury. Cochrane Database Syst Rev 2017;20(1). https://doi.org/10.1002/14651858.CD006787.pub3

23. Mainka S, Wissel J, Völler H, Evers S. The use of rhythmic auditory stimulation to optimize treadmill training for stroke patients: A randomized controlled trial. Front Neurol 2018;9:755. https://doi.org/10.3389/fneur.2018.00755

24. Moumdjian L, Sarkamo, Leone C, Leman M, Feys P. Effectiveness of music-based interventions on motricity or cognitive functioning in neurological populations: a systematic review. Eur J Phys Rehabil Med 2017;53(3):466-82. https://doi.org/10.23736/S1973-9087.16.04429-4

25. Orantin M, Yelnik A, Jousse M, Guillemette M, Bernard A, Tlili L, Quintaine V. Give music therapy a chance in post-stroke rehabilitation. Ann Phys Rehabil Med 2018;61(6):479-20. https://doi.org/10.1016/j.rehab.2018.01.004

26. Pohl P, Carlsson G, Bunketorp Käll L, Nilsson M, Blomstrand C. Experiences from a multimodal rhythm and music-based rehabilitation program in late phase of stroke recovery - A qualitative study. PLoS One 2018;13(9). https://doi.org/10.1371/journal.pone.0204215

27. Street AJ, Magee WL, Bateman A, Parker M, Odell-Miller H, Fachner J. Home-based neurologic music therapy for arm hemiparesis following stroke: results from a pilot, feasibility randomized controlled trial. Clin Rehabil 2018;32(1):18-28. https://doi.org/10.1177/0269215517717060 